\title{
Long-Term Application of Cerebellar Transcranial Direct Current Stimulation Does Not Improve Motor Learning in Parkinson's Disease
}

\author{
Lidio Lima de Albuquerque ${ }^{1} \cdot$ Milan Pantovic $^{2} \cdot$ Mitchell G. Clingo $^{3} \cdot$ Katherine M. Fischer $^{2} \cdot$ Sharon Jalene $^{2}$. \\ Merrill R. Landers ${ }^{4} \cdot$ Zoltan Mari $^{5} \cdot$ Brach Poston $^{2}$ (1)
}

Accepted: 18 June 2021 / Published online: 7 July 2021

(c) The Author(s), under exclusive licence to Springer Science+Business Media, LLC, part of Springer Nature 2021

\begin{abstract}
Cerebellar transcranial direct current stimulation (c-tDCS) enhances motor skill acquisition and motor learning in young and old adults. Since the cerebellum is involved in the pathophysiology of Parkinson's disease (PD), c-tDCS may represent an intervention with potential to improve motor learning in PD. The primary purpose was to determine the influence of long-term application of c-tDCS on motor learning in PD. The secondary purpose was to examine the influence of long-term application of c-tDCS on transfer of motor learning in PD. The study was a randomized, double-blind, SHAM-controlled, between-subjects design. Twenty-one participants with PD were allocated to either a tDCS group or a SHAM stimulation group. Participants completed 9 practice sessions over a 2-week period that involved extensive practice of an isometric pinch grip task (PGT) and a rapid arm movement task (AMT). These practice tasks were performed over a 25-min period concurrent with either anodal c-tDCS or SHAM stimulation. A set of transfer tasks that included clinical rating scales, manual dexterity tests, and lower extremity assessments were quantified in Test sessions at Baseline, 1, 14, and 28 days after the end of practice (EOP). There were no significant differences between the c-tDCS and SHAM groups as indicated by performance changes in the practice and transfer tasks from Baseline to the 3 EOP Tests. The findings indicate that long-term application of c-tDCS does not improve motor learning or transfer of motor learning to a greater extent than practice alone in PD.
\end{abstract}

Keywords Parkinson's disease · Transcranial direct current stimulation $\cdot$ Motor skill $\cdot$ Cerebellum $\cdot$ Cerebellar stimulation

\section{Introduction}

Parkinson's disease (PD) is the second most common neurodegenerative disorder after Alzheimer's disease [1]. It is characterized by progressive dopaminergic cell loss in the substantia nigra, which results in a reduction of dopamine in the striatum [2]. These underlying physiological mechanisms

Brach Poston

brach.poston@unlv.edu

1 School of Health and Applied Human Sciences, University of North Carolina at Wilmington, Wilmington, NC, USA

2 Department of Kinesiology and Nutrition Sciences, University of Nevada Las Vegas, Las Vegas, NV, USA

3 School of Medicine, University of Nevada Las Vegas, Las Vegas, NV, USA

4 Department of Physical Therapy, University of Nevada Las Vegas, Las Vegas, NV, USA

5 Movement Disorders Program, Cleveland Clinic Lou Ruvo Center for Brain Health, Las Vegas, NV, USA lead to a number of motor impairments such as bradykinesia, rigidity, tremor, and postural instability that severely limit the capability of individuals with PD to accomplish many essential daily living activities [3, 4]. Although current pharmacological, surgical, and physical exercise treatment approaches are valuable, they are also associated with limitations such as moderate effectiveness, excessive costs, and an array of side effects. Therefore, development of new adjunct interventions that are effective and have a realistic potential to be implemented into clinical practice are needed in the treatment of PD [5].

Transcranial direct current stimulation (t-DCS) could represent one such intervention that could satisfy many of these requirements [6]. Most commonly, tDCS is delivered to the motor cortex (M1-tDCS) and has been shown to enhance motor skill acquisition and learning in a variety of populations including PD. Accordingly, the majority of tDCS studies have reported motor performance enhancements of $10-15 \%$ during or shortly after a single application lasting 10-20 min when compared to practice alone in young adults 
and old adults [7] as well as in PD [8-10]. Most importantly, longer-term studies lasting between 3 and 10 days have demonstrated that these improvements in performance can be increased to up to twice the magnitude attained in a single session in healthy adults [11-13] and in one notable study in PD [14]. Despite these positive findings involving M1-tDCS in PD, development of new non-invasive brain stimulation techniques or the targeting of additional brain areas could provide additional avenues to improve motor skill acquisition and learning in PD. Recently, tDCS delivered to the cerebellum (c-tDCS) has also been reported to significant improve motor skill in young [15-19] and old adults [20]. The ability of c-tDCS to impact motor skill learning in older adults is particularly interesting because accumulating evidence suggests that the cerebellum may be the primary brain area responsible for the movement impairments often observed in older adults [21], and most individuals with PD are older adults.

In addition to the positive effects of c-tDCS on motor performance in heathy adults, several other lines of evidence provide basis for utilizing c-tDCS in PD: 1) the cerebellum contributes to the motor deficits of PD [22, 23]; 2) bidirectional pathways between cerebellum and basal ganglia were recently identified [24], which is particularly relevant because tDCS can induce physiological changes in deeper and interconnected brain areas not directly stimulated. For example, M1-tDCS applied to PD monkeys impacted basal ganglia function and improved motor performance [25]; and 3) cerebellar dysfunction may be a compensatory mechanism in PD in an attempt to mitigate the negative influences of abnormal basal ganglia activity. Accordingly, people with PD that have greater compared to lower cerebellar activity display better motor performance [23]. Therefore, c-tDCS could potentially increase motor skill in PD by enhancing these compensatory processes via increasing cerebellar activity [26].

The contributions of the cerebellum to distinct motor control processes and motor learning provide further rationale for the investigation of c-tDCS in PD. For example, the cerebellum is highly involved in the control of visual guided movements, multi-joint movements that involve joint interaction torque regulation and complex agonist-antagonist muscle coordination patterns, and error detection in goaldirected movements. The cerebellum also plays a major role in specific aspects of motor learning such as motor adaptation learning (modifying motor skills to new environments) [27], procedural learning (automatic, non-conscious processes) [28], and consolidation of learned movements [29]. Most importantly, numerous studies have shown cerebellar involvement in both short-term and long-term motor skill learning, depending on the details of the motor task and the experimental conditions [30]. These processes occur in healthy populations and in PD; however, individuals with
PD display impairments in all of these aspects of motor learning [31-33], which could be at least partially associated with cerebellar dysfunction [23]. Since the cerebellum displays notable intrinsic plastic changes during motor learning and tDCS is known to elicit and heighten plasticity [29], $\mathrm{c}$-tDCS could enhance motor learning in PD. Despite these interrelated observations, it is surprising that no long-term studies have examined the influence of c-tDCS on motor learning in PD.

The primary purpose was to determine the influence of long-term application of c-tDCS on motor learning in PD. The secondary purpose was to examine the influence of long-term application of c-tDCS on transfer of motor learning in PD. This was accomplished by requiring two groups of PD patients to perform two practice tasks simultaneous with administration of c-tDCS or SHAM stimulation over the course of 9 practice sessions. Motor performance on the same practice tasks was assessed on these same tasks in 4 test sessions performed before (Baseline) and 1, 14, and 28 days after the practice and stimulation sessions ceased. In addition, transfer tasks were completed in the test sessions to quantify transfer of motor learning to tasks not practiced extensively or performed during c-tDCS. It was hypothesized that c-tDCS would enhance motor learning and transfer of motor learning to a greater extent than practice alone in PD. These predictions were based on the previous c-tDCS studies in young and old adults [15-20] and M1-tDCS studies in PD [8-10, 14, 34, 35] that collectively observed significant enhancements in motor skill due to the administration of tDCS.

\section{Materials and Methods}

\section{Participants}

A total of 21 individuals with PD (11 males, 10 females; mean age: $71.2 \pm 8.9$ ) volunteered to participate in the study and provided written informed consent. Additional participant demographic information and clinical characteristics are provided in Table 1 and a flow diagram of the participants through the phases of the study is depicted in Fig. 1. All participants were clinically diagnosed with idiopathic PD, free of any other neurological disorder, did not meet international non-invasive brain stimulation exclusion criteria, and had no uncontrolled medical conditions. The handedness of each participant was determined using the Edinburgh Handedness Inventory [36]. All participants performed the experiments with the dominant hand/arm, which was also their primarily affected side. A total of 19 of the participants were primarily right-side affected and right-hand dominant and 2 participants were primarily 
Table 1 Participant demographic information and clinical characteristics

\begin{tabular}{llll}
\hline & All & c-tDCS & SHAM \\
\hline Number of Participants & 21 & 11 & 10 \\
Age, mean (SD) & $71.2(8.9)$ & $70.1(7.1)$ & $71.5(10.9)$ \\
Hoehn and Yahr stage, mean (SD) & $2.1(0.7)$ & $2.3(0.6)$ & $2.0(0.7)$ \\
LEDD (mg), mean (SD) & $519.1(389.2)$ & $549.8(516.2)$ & $485.4(195.5)$ \\
Right-side affected and right-hand dominant & 19 & 10 & 9 \\
Left-side affected and Left-hand dominant & 2 & 1 & 1
\end{tabular}

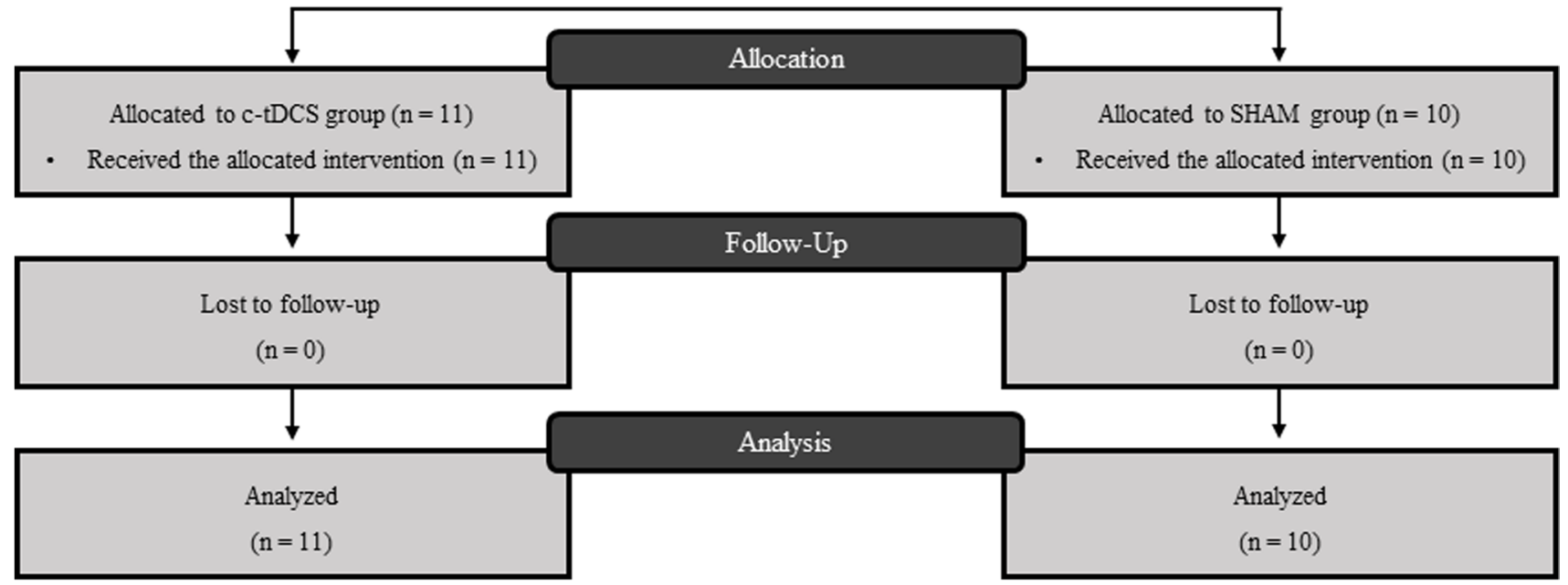

Fig. 1 Participant flow diagram of the progress of participants through the enrollment, intervention allocation, follow-up, and data analysis phases of the study

left-side affected and left-hand dominant. All procedures were approved by the University of Nevada Las Vegas institutional review board and were conducted according to the Declaration of Helsinki.

\section{Experimental Design}

The study was a randomized, double-blind, SHAM-controlled, between-subjects design. A schematic of the overall experimental design and schedule is depicted in Fig. 2A.
Participants were randomized into either the c-tDCS or SHAM group (Research Randomizer; www. randomizer.org) by an investigator who did not participate in data collection or data analysis but programmed the stimulator in each session. Therefore, the investigators who collected and analyzed data were blinded to the experimental conditions (see c-tDCS section below). The study had an overall duration of 40 days. On Day 1, participants completed the Baseline test session followed by the first practice session. Subsequently, 4 consecutive identical practice sessions were performed 
A

Baseline

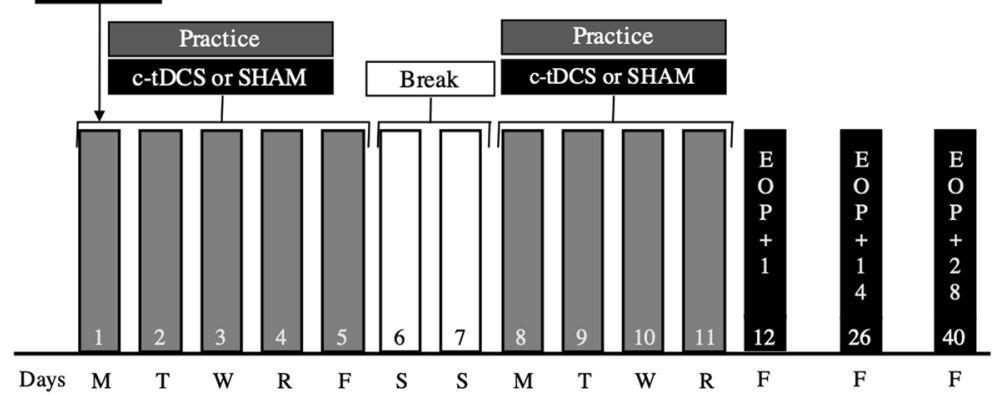

B

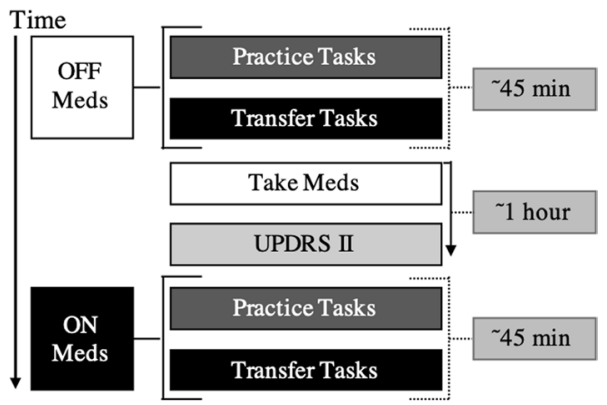

Fig. 2 Schematic representation of the experimental schedule, design, and protocol. A. Participants completed 4 test sessions that included a Baseline session on Day 1 and three end of training (EOT) test sessions at 1,14 , and 28 days $(\mathrm{EOT}+1, \mathrm{EOT}+14, \mathrm{EOT}+28)$ after the last practice day. Accordingly, at total of 9 practice sessions were performed over a 2-week practice period comprising 5 practices sessions on weekdays of the first week and 4 practices sessions on weekdays of the second week (the letters on the $x$ axis denote the days of the

followed by a weekend break of 2 days. Next, 4 more consecutive weekday practice sessions were conducted in the same manner as before. Finally, 3 end of training (EOT) test sessions were completed at 1,14 , and 28 days $(\mathrm{EOT}+1$, $\mathrm{EOT}+14, \mathrm{EOT}+28)$ after the last practice day.

\section{Test Sessions}

These sessions were conducted in the same manner on all 4 occasions and the following procedures were performed in the order prescribed: 1) participants reported to the lab in the morning after a 12-h overnight medication withdrawal (practically defined-OFF State) [37]. Participants were tested in the OFF state to provide information on the effects of c-tDCS on the basic pathology of PD in the relative absence of medication influences; 2) the practice tasks (PGT and AMT) were performed for 10 trials and 1 block of 20 trials, respectively; 3) the Unified Parkinson's Disease Rating Scale Part III (UPDRS-III), Purdue Pegboard Test (PPT), and Jebsen Taylor Hand Function Test (JTT) were administered; 4) the lower extremity tests (gait and balance tests) were completed; 5) the participants ingested their medications, filled out the UPDRS II, and rested quietly for one hour allowing the medications to take effect; 6 ) steps $2-4$ were repeated while the patients were on medications (ON state).

\section{Practice Sessions}

Nine practice sessions were performed over period of 2 weeks (Week 1: 5 sessions, Mon-Fri; Week 2: 4 sessions, Mon-Thu; Fig. 2) Each practice session was administered in an identical manner on all 9 practice days according to the following procedures in the order described: 1) participants week). Participants performed 2 practice tasks with their dominant, primarily affected hand/arm each of the 9 practice sessions simultaneous with administration of c-tDCS. B. A single test session involved performing the practice tasks (no-c-tDCS) followed by the transfer tasks in the OFF state. Subsequently, participants ingested their medications, rested for $1 \mathrm{~h}$, and repeated the practice task and transfer task testing in the $\mathrm{ON}$ state

reported to the lab in the morning in the ON medication state; 2$)$ the c-tDCS electrode montage was placed on the participant; 3) the stimulator was turned on for $3 \mathrm{~min}$ while participants sat quietly before starting the first PGT trial [18]; 4) 10 trials of the PGT were performed; 5) the AMT task was executed for 4 blocks of 20 trials; and 6) the stimulator was kept on after completion of the last AMT trial block, which usually involved a time period of 1-3 min until the 25-min stimulation period elapsed and the stimulator turned off. Thus, in each of these practice days, the PGT and AMT practice were performed simultaneous with administration of c-tDCS over a practice period of approximately $20 \mathrm{~min}$. Importantly, it is crucial to emphasize that the practice sessions were performed while patients were in the ON state. The reasoning for this was that for c-tDCS to be a viable intervention in $\mathrm{PD}$, it would need to be able to produce improvements in motor performance while patients are in the $\mathrm{ON}$ state for real world application.

\section{Practice Tasks}

The two practice tasks comprised the PGT and the AMT and both tasks were performed during both the test sessions (no c-tDCS applied) and the practice sessions (concurrent with tDCS). This design allowed for the quantification of motor learning across both the test sessions and the practice sessions.

\section{PGT}

The PGT was considered the primary practice task because similar isometric precision grip force tasks have been used in several long-term tDCS studies [11-13, 16, 38]. The 
experimental arrangement was organized in a similar manner to prior studies [39, 40]. Briefly, participants sat in a chair with a table positioned to the side of the chair corresponding to their dominant, primarily affected hand/arm. A computer monitor was located directly in front of the participant a meter away at eye level and provided all visual feedback for the task. The posture assumed by the participant was as follows: 1) the forearm was placed on the table and the wrist was in neutral and the hand semi-supinated; 2) the shoulder was abducted to $\sim 45^{\circ}$; and 3 ) the elbow was flexed to $\sim 90^{\circ}$.

The PGT involved attempting to match a target sine wave $(1 \mathrm{~Hz})$ template by producing isometric force using a precision grip (index finger and thumb) against a grip device. This grip device comprised a custom-made manipulandum that was embedded with separate force transducers for each digit. The sine wave target template was displayed on the monitor and scrolled across the screen with time. To match the target template, the participants had to attempt to trace the template by producing an appropriate force-time profile. Thus, the participants were required to control the sum of the index finger and digit forces (total force), which was displayed on screen in combination with the template and to match the target template as accurately as possible throughout each PGT trial. The minimums and maximums of the target sine wave were 5\% and 35\% of the precision grip maximum voluntary contraction force (MVC) of the same PGT task. This MVC value was determined in the first test session using previously described methods [41]. Thus, the MVC value in the first test session was used to determine the PGT target force values and this target force was kept the same over the course of the 9 practice sessions. Finally, each PGT trial involved matching the target template for $30 \mathrm{~s}$ followed by a 30 -s rest period.

This PGT task was chosen as the primary practice task and to be paired with c-tDCS for the following reasons: 1) neural control of precision grip tasks have been well-characterized in healthy participants, older adults [42, 43], and in PD [44]; 2) the PGT parameters can be made sufficiently difficult enough so that performance has the potential for continual improvement [11-13] over most of the 9 practice sessions and due to c-tDCS; 3 ) the precision grip is a complex, functional task required extensively in everyday living that involves many muscles and a widely-distributed cortical network; 4) there is strong cerebellar involvement in visuomotor tracking tasks [45]; and (5) a precision grip task variant was employed in long-term M1-tDCS [11-13] and a c-tDCS study in young adults [16].

\section{AMT}

The AMT was considered the secondary practice task because it has not been used as extensively in the most relevant tDCS studies. However, variations of arm movement tasks like the AMT have been performed in numerous motor control studies in a wide range of populations. In addition, arm movement task performance and deficits are very well-characterized in PD [46]. The AMT was conducted using almost identical methodology to a previous study in healthy young and old adults [47]. Briefly, participants performed the AMT with the dominant, primarily affected hand/arm on a digitizer tablet with a digitizer pen. Participants were directed to execute the AMT as fast and as accurately as possible using a single, uncorrected movement that required elbow extension and shoulder flexion from a home circle $(1.5 \mathrm{~cm}$ diameter $)$ to a very small target circle $(0.5 \mathrm{~cm}$ diameter) located $20 \mathrm{~cm}$ away in a straight line. The sequence of task events was implemented by a customized data acquisition script and visual feedback of the task was provided on a computer monitor linked to the tablet. Once participants had attained the correct starting position in the home circle, a "GO" signal was presented. Participants then executed the movement at their own convenience (no reaction time component). Visual feedback of the cursor movement (trajectory) was not provided to participants during the trials. However, they received visual feedback of their final endpoint position relative to the target in the form of a small dot after every trial for a time period of $3 \mathrm{~s}$. Finally, participants were told to continually try to minimize their endpoint error on each successive trial.

This AMT was chosen as a practice task and paired with c-tDCS for the following reasons: 1 ) there is strong cerebellar involvement in complex, multi-joint arm movement tasks that involves the prediction, exploitation, and compensation for the effects of joint interaction torques [48, 49]; 2) the cerebellum is highly involved in the timing of activation of antagonistic muscle groups [50]; and 3) the cerebellum is implicated in error detection in goal-directed movements [51]. Accordingly, all of these features of the neural control of movement are present in the execution of the AMT.

\section{Transfer Tasks}

A total of 8 transfer tasks were utilized to quantify transfer of motor learning to tasks that were not executed simultaneously with c-tDCS application and were not practiced nearly as extensively as the practice tasks. This was due to the fact that they were only performed in the test sessions for 1-3 trials each. The transfer tasks were comprised of 2 clinical rating scales, 2 manual dexterity tests, and 4 lower extremity tests. The manual dexterity tests were considered near transfer tasks as they most closely resembled the muscles of the hand-arm system compared to the practice tasks, whereas the other 6 transfer tasks were considered far transfer tasks as the clinical rating scales only partially involve upper limb 
movements and the lower extremity tasks involve leg muscles and movements.

\section{Transfer Tasks-Clinical Rating Scales}

The clinical ratings scales included the UPDRS-III and UPDRS II. The UPDRS-III was completed on the test days in both the ON and OFF states. In contrast, the UPDRS II was completed only once on each test day (see below). The UPDRS-III was administered by an investigator trained by a movement disorders neurologist. The UPDRS-III is the gold standard clinical test to evaluate motor and symptoms in PD. The UPDRS-II is a self-evaluation survey to assess the performance of daily living activities over the prior week. Thus, it could potentially measure the degree of global improvements elicited by c-tDCS. In contrast to all the other transfer tests, it was only administered once in each testing period since it is a questionnaire which involves questions regarding the entire previous week making $\mathrm{ON}$ and OFF state testing inapplicable compared to the other transfer tests.

\section{Transfer Tasks—Manual Dexterity Tests}

The manual dexterity tasks were completed on the test days in both the ON and OFF states and included the PPT and the JTT. The PTT is a standard test to assess multi-joint arm and hand dexterity. One trial of the task involves picking up small pegs from a bowl-shaped tray and inserting as many as possible over a time period of $30 \mathrm{~s}$ into a column of holes as fast as possible. The JTT is a well-validated test that has commonly used to measure functional manual dexterity in aging, movement disorders, and tDCS experiments [52]. It comprises the performance of six tasks that mimic customary activities of daily living including: flipping cards, handling small objects, feeding, and the stacking and manipulation of cans. For each of the tasks, the time taken to complete them measured by one of the investigators.

\section{Transfer Tasks—Lower Extremity Tests}

The lower extremity tests were administered on the test days in both the ON and OFF states and comprised three measures of gait performance (step length, step length coefficient of variation (CV), gait velocity) and one measure of balance performance (balance composite score). Gait kinematics were measured with a pressure sensor-engineered walking mat (Protokinetics ${ }^{\ddagger}$, Havertown, PA). Participants were asked to walk across the 20 -foot mat using their normal gait with the first step of each trial utilizing the right leg. Balance was assessed using the Bertec Balance System Sensory Organization Test (SOT). This test involves quantification of the postural sway of the participant while they stand in a standardized static position. A total of six different conditions are assessed with each condition involving visual and mechanical perturbations or a combination of both for 20-s trials. The system outputs the algorithmcalculated scores for each of the six conditions as well as a balance composite score and sensory scores for the three balance sensory systems (i.e., visual, vestibular, and somatosensory). The balance composite score was selected as a measure of overall balance performance. Although it could also be viewed as counterintuitive that the lower extremity tasks could be modulated with the same c-tDCS parameters as upper limb tasks. However, one study [19] used the same c-tDCS parameters as in upper limb c-tDCS studies and found an increase in locomotor adaptation rates.

\section{c-tDCS}

Anodal c-tDCS was delivered with a NeuroConn DC Stimulator Plus/MR via two rubber electrodes $(5 \times 5 \mathrm{~cm})$ that were encased in saline soaked sponges. Accordingly, the anode was placed $3 \mathrm{~cm}$ lateral to the inion over the cerebellum ipsilateral to the primarily affected hand and the cathode was placed over the ipsilateral buccinator muscle. The anode was positioned ipsilateral to the primarily affected hand because outputs from the cerebellar hemisphere on one side of the body cross the midline and act on the contralateral M1. Since the vast majority of M1 outputs also cross the midline and act on contralateral motor neurons, the cerebellar hemisphere on one side of the body influences the hand/arm system on the same side (ipsilateral) of the body. Thus, the main goal was to enhance performance in the most affected limb. The current strength was $2 \mathrm{~mA}$ and the stimulation duration was $25 \mathrm{~min}$. Collectively, these c-tDCS parameters have elicited significant motor performance enhancements in numerous previous studies in young and old adults by another research group [15-17, 19, 20,53] and in a previous study in our lab in young adults [18]. Based on these studies and the mixed success of other c-tDCS studies that used different parameters, we thought that these parameters were the most likely to be successful and would be the most reasonable starting point for PD experiments. For SHAM stimulation, the current was ramped up and down over $30 \mathrm{~s}$ according to the most commonly accepted protocol [6]. An investigator who did not participate in data collection or analysis programmed the stimulator in each session. Finally, the investigators who conducted the experiments and analyzed the data were also blinded to the experimental conditions experienced by the participants.

\section{Data Analysis}

All PGT data were collected and analyzed in customwritten scripts in Spike2 software (CED, Cambridge UK), whereas AMT data were collected using Movalyzer software 
(Neuroscript; Tempe, Arizona) and analyzed in custom-written Matlab scripts according to previous methods [47]. For the PGT, performance was quantified as the average error in force relative to the target force template over each 30-s trial. More specifically, the absolute value of the difference at each sampling point between the force template and the force produced by that participant was quantified and then averaged over the entire 30 -s trial. The average of the 5 trials in each test session and 10 trials in each practice session (grand averages) were taken as the final force error values for analysis. The endpoint accuracy in the AMT was quantified as the endpoint error [18, 47, 54] using the Pythagorean Theorem. Thus, endpoint error was calculated as the shortest absolute distance between the target center's $x, y$ coordinates and the final endpoint $x, y$ coordinates of the digitizer pen trajectory (for a detailed description of all the associated calculations see Poston et al. [47]). The average of the 20 trials in each test session block was taken as the final endpoint values for analysis for the test sessions. In contrast, the average endpoint error for the 4 blocks of 20 trials performed on practice days was taken as the final endpoint error values for analysis for each practice day. The UPDRS-III score was quantified as the sum of the items (scored on a 5-point scale) and used for all analysis. Similarly, the UPDRS-II score was also simply the sum of all the items associated with the questionnaire. The PPT score was quantified as the average number of pegs placed in the holes within a $30 \mathrm{~s}$ trial with the average of the 3 trials being used for analysis. For the JTT, time to complete each of the 6 tasks was computed for each trial and the sum of these times was denoted as the total time for a trial. Finally, the average of the 3 trials was computed. The gait variables of step length, step length $\mathrm{CV}$, and gait velocity were quantified by Protokinetics software using the center of pressure data. A total of three passes were made across the mat and the average values for these passes were used for analysis. For the balance testing, the six balance conditions were used by the Bertec system algorithm to calculate the balance composite score for analysis.

\section{Statistical Analysis}

An a priori power analysis was conducted to determine the number of participants required using data from a previous M1-tDCS study in PD [35]. Specifically, we used extrapolations of UPRDRS III data from Fregni et al. (2006), to estimate effects sizes (Cohen's D $>1.0$ ), which were then used for sample size calculations. A sample of 12 participants (including an estimated 10\% dropout rate based on previous studies in our lab) would be sufficient to achieve $>80 \%$ power at $\alpha=0.05$ using the "paired t-test using effect size" module on PASS 2020 (NCSS, LLC. Kaysville, Utah, USA, www.ncss.com/software/pass).
After the completion of 21 participants, the Covid-19 pandemic necessitated that we stop recruitment. Since it was anticipated that recruitment would not be allowed under university pandemic restrictions for the near future, we decided to re-evaluate the trial approach and do an interim analysis to estimate the sample size needed for the three primary dependent variables (PPT, AMT, UPDRS-III) for the test sessions. Thus, this interim futility analysis approach was used to determine if additional resources and recruitment were needed once the pandemic restrictions were lifted. Using the means and standard deviations from these analyses and the "repeated measures analysis" module on PASS 2020 , it was determined that 284 participants (263 additional participants) would be needed to achieve sufficient power to find statistically significant interactions for the PPT. Furthermore, identical analysis for the AMT and UPDRS-III determined that 619 participants (598 additional participants) and 113 participants (92 additional participants) would be needed for statistically significant interactions, respectively. Based on the impracticality of recruiting these participants and the very small observed effect sizes, we decided to terminate the study early for futility because there was no apparent treatment effect.

The demographic and clinical characteristic data of age and levodopa equivalent daily dose (LEDD) were compared between the two groups with unpaired $t$-tests, whereas Hoehn and Yahr scores were compared with a Chi Square Test. For the test sessions, all dependent variables except for the UPDRS-II were analyzed with three-way mixed ANOVAs: 2 group $(\mathrm{c}-\mathrm{tDCS}, \mathrm{SHAM}) \times 4$ test (Baseline, $\mathrm{EOP}+1, \mathrm{EOP}+14, \mathrm{EOP}+28) \times 2$ condition $(\mathrm{ON}$ state, OFF state). The UPDRS-II was analyzed with a two-way mixed ANOVA: 2 group $(\mathrm{c}-\mathrm{tDCS}, \mathrm{SHAM}) \times 4$ test (Baseline, $\mathrm{EOP}+1, \mathrm{EOP}+14, \mathrm{EOP}+28)$. Post hoc comparisons using the Bonferroni adjustment for multiple comparisons were performed to locate significant differences when appropriate. For the practice sessions, the percent change in force error (PGT) and endpoint error (AMT) from Practice Day 1 to Practice Day 9 between groups was compared with two-tailed unpaired $t$-tests. The significance level was set at $\alpha<0.05$ and data are indicated as means \pm standard errors in the figures.

\section{Results}

\section{Demographic and Clinical Characteristics}

The age of the participants, LEDD, and Hoehn and Yahr stage were non-statistically significant between the c-tDCS and SHAM groups $(P=0.883, P=0.715$, and $P=0.273$, respectfully; Table 1). 


\section{Test Sessions}

Practice Tasks For the force error in the PGT, the group $\times$ test $\times$ condition $\left(F_{(3,57)}=0.120, P=0.948, \eta^{2}=0.006\right)$, group $\times$ test $\left(F_{(3,57)}=0.328, P=0.805, \eta^{2}=0.017\right)$, and group $\times$ condition $\left(\mathrm{F}_{(1,19)}=0.328, P=0.805, \eta^{2}=0.017\right)$ interactions were all non-statistically significant. However, there was a significant test $\times$ condition interaction $\left(F_{(3,57)}=\right.$ $\left.3.33, P=0.026, \eta^{2}=0.149\right)$ and post hoc analyses of the interaction indicated that force error was significantly lower in the ON compared with the OFF state at EOP+1, EOP+14, and EOP+28 (all $P$ values $<0.001$; Fig. 3A). According, there was also a significant main effect for test $\left(F_{(3,57)}=\right.$ $\left.41.547, P=0.001, \eta^{2}=0.686\right)$ and post hoc analyses indicated that force error was significantly lower at EOP+1, $\mathrm{EOP}+14$, and EOP +28 compared to Baseline (all $P$ values below < 0.001). Finally, the main effect for group $\left(\mathrm{F}_{(1,19)}=\right.$ $\left.0.350, P=0.561, \eta^{2}=0.018\right)$ and main effect for condition $\left(F_{(1,19)}=0.714, P=0.409, \eta^{2}=0.036\right)$ were not significant.
For endpoint error in the AMT, the group $\times$ test $\times$ condition $\left(F_{(3,51)}=0.662, P=0.579, \eta^{2}=0.037\right)$, group $\times$ test $\left(F_{(3,51)}=0.473, P=0.703, \eta^{2}=0.027\right)$, test $\times$ condition $\left(F_{(3,51)}=0.651, P=0.586, \eta^{2}=0.037\right)$, and group $\times$ condi tion $\left(F_{(3,51)}=2.270, P=0.150, \eta^{2}=0.118\right)$ interactions were all non-statistically significant. In addition, the main effect for $\operatorname{group}\left(F_{(1,17)}=0.064, P=0.803, \eta^{2}=0.004\right)$ and main effect for condition $\left(F_{(1,17)}=0.642, P=0.434, \eta^{2}=0.036\right)$ were not statistically significant. However, there was a significant main effect $\left(F_{(3,51)}=4.900, P=0.005, \eta^{2}=0.224\right)$ for test and post hoc analyses indicated that endpoint error was significantly lower at EOP +1 , and EOP +28 compared to Baseline (all $P$ values $<0.001$; Fig. 3C).

\section{Transfer Tasks_-Clinical Rating Scales}

For the UPDRS-III scores, the group $\times$ test $\times$ cond $i$ tion $\left(F_{(3,57)}=1.171, P=0.163, \eta^{2}=0.085\right)$, group $\times$ test $\left(F_{(3,57)}=1.990, P=0.126, \eta^{2}=0.095\right)$, test $\times$ condition $\left(F_{(3,57)}=0.351, P=0.789, \eta^{2}=0.018\right)$, and group $\times$ condition $\left(F_{(1,19)}=0.233, P=0.635, \eta^{2}=0.012\right)$ interactions
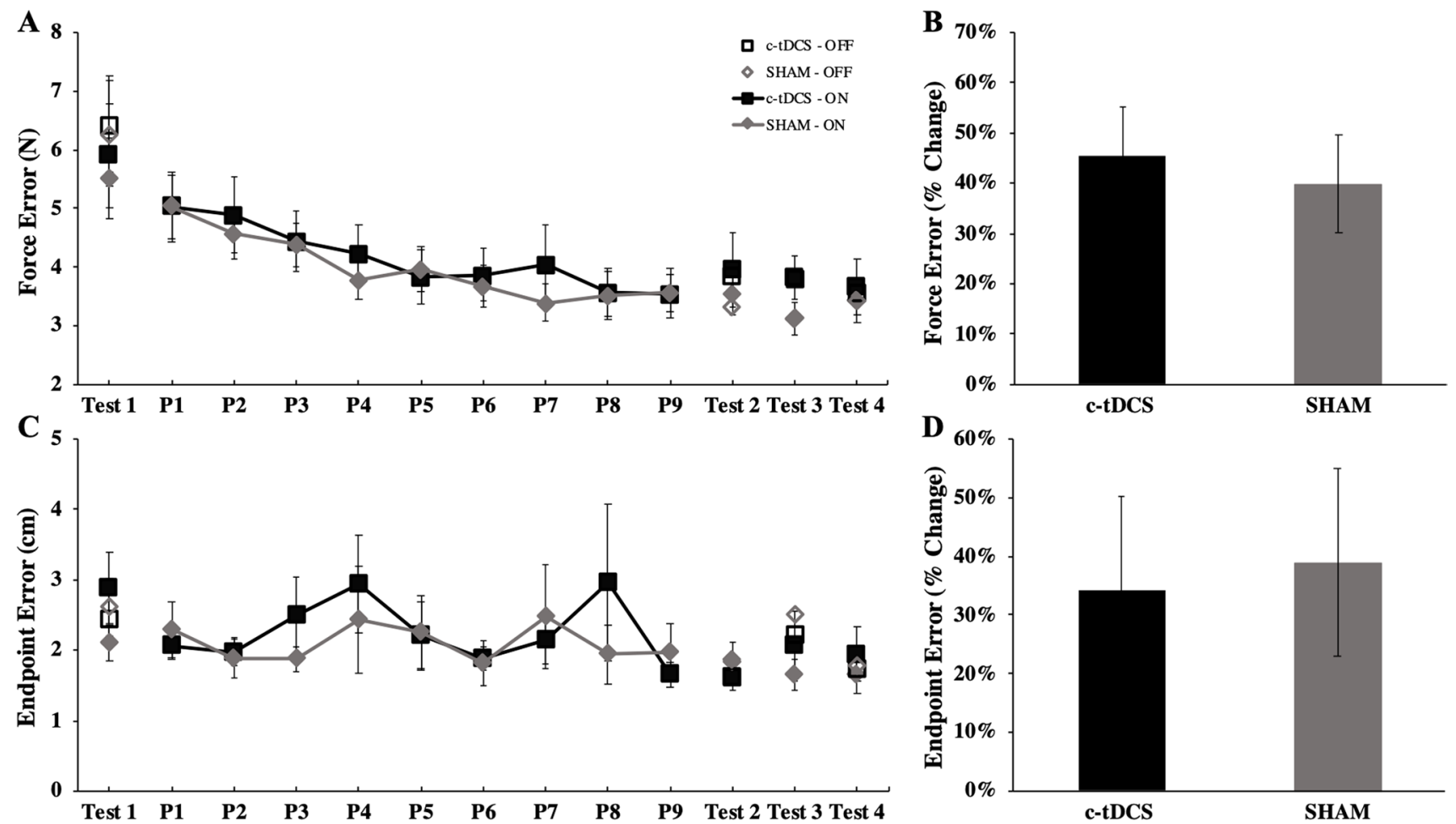

Fig. 3 Force error in the PGT and endpoint error in the AMT in the test and practice sessions for the c-tDCS and SHAM groups in the ON and OFF states. A. Force error declined across the test sessions $(P=0.001)$, but the reduction in force error was similar for the $\mathrm{c}-\mathrm{tDCS}$ and SHAM groups $(P=0.561)$. The force error was also similar in the ON and OFF states in the test sessions $(P=0.409)$. B. The percent change in force error (decline) from Practice session 1 to Practice session 9 was similar for the c-tDCS and SHAM groups
$(P=0.698)$. C. Endpoint error declined across the test sessions $(P=0.005)$, but the reduction in endpoint error was similar for the c-tDCS and SHAM groups $(P=0.803)$. The endpoint error was also similar in the ON and OFF states in the test sessions $(P=0.409)$. D. The percent change in endpoint error (decline) from Practice session 1 to Practice session 9 was similar for the c-tDCS and SHAM groups $(P=0.860)$ 
were all non-statistically significant. In addition, the main effect for $\operatorname{group}\left(F_{(1,19)}=0.131, P=0.721, \eta^{2}=0.007\right)$ and main effect for test $\left(F_{(3,57)}=0.982, P=0.408, \eta^{2}=0.049\right)$ were not significant. However, there was a significant main effect $\left(F_{(1,19)}=43.078, P=0.001, \eta^{2}=0.694\right)$ for condition and post hoc analyses indicated that UPDRS-III scores were significantly higher in the OFF state compared to the ON state (Fig. 4A). For the UPDRS-II scores, the group $\times$ test $\left(F_{(3,57)}=3.916, P=0.013, \eta^{2}=0.171\right)$ interaction was statistically significant (Fig. 4B) and post hoc analyses indicated that UPDRS-II scores were lower for the c-tDCS group at $\mathrm{EOP}+14$ and $\mathrm{EOP}+28(P=0.002$, and 0.004 , respectively $)$. In addition, there was a significant main effect for test $\left(F_{(3,57)}=7.198, P=0.001, \eta^{2}=0.275\right)$ and post hoc analyses indicated that UPDRS-II scores were significantly lower at $\mathrm{EOP}+14$, and $\mathrm{EOP}+28$ compared to baseline $(P=0.001$ and 0.006 , respectively). Finally, the main effect for group was not statistically significant $\left(F_{(1,19)}=0.231, P=0.636\right.$, $\eta^{2}=0.012$ ).
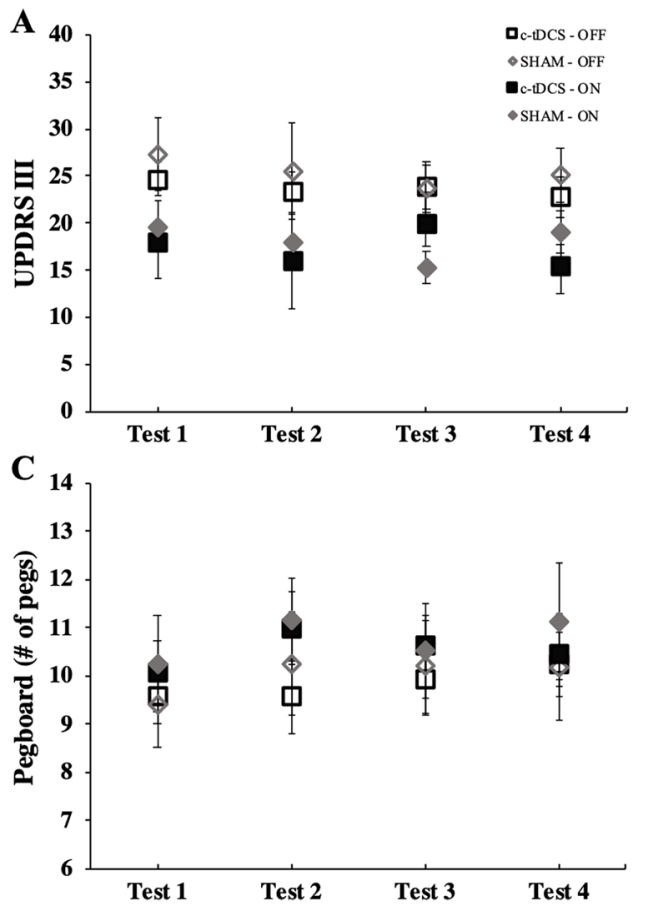

Fig. 4 UPDRS-III, PPT, and JTT scores in the test sessions for the c-tDCS and SHAM groups in the ON and OFF states along with UPDRS-II scores in the test sessions A. UPDRS-III scores were similar for the c-tDCS and SHAM groups $(P=0.721)$ and did not change across the test sessions $(P=0.408)$. However, UPDRS-III scores were significantly higher in the OFF state compared to the ON state $(P=0.001)$. B. UPDRS-II scores were similar for the c-tDCS and SHAM groups $(P=0.636)$ and were lower at $\mathrm{EOP}+14$ and $\mathrm{EOP}+28$ compared to baseline $(P=0.001$ and 0.006 , respectively). C. PPT

\section{Transfer Tasks—Manual Dexterity Tests}

For the PPT, the group $\times$ test $\times$ condition $\left(F_{(3,57)}=1.384\right.$, $\left.P=0.257, \eta^{2}=0.068\right)$, group $\times$ test $\left(F_{(3,57)}=0.440, P=0.725\right.$, $\left.\eta^{2}=0.023\right)$, test $\times$ condition $\left(F_{(3,57)}=1.289, P=0.227\right.$, $\left.\eta^{2}=0.073\right)$, and group $\times$ condition $\left(F_{(1,19)}=0.004, P=0.953\right.$, $\left.\eta^{2}=0.001\right)$ interactions were all non-statistically significant. In addition, the main effect for $\operatorname{group}\left(F_{(1,19)}=0.033\right.$, $P=0.857, \eta^{2}=0.002$ ) was not statistically significant. However, there was a significant main effect for condition $\left(F_{(1,19)}=13.289, P=0.002, \eta^{2}=0.415\right)$, which indicated that the number of pegs was significantly greater in the $\mathrm{ON}$ state compared to the OFF state (Fig. 4C). There was also a significant main effect for test $\left(F_{(3,57)}=4.668, P=0.006\right.$, $\left.\eta^{2}=0.197\right)$ and post hoc analyses indicated that the number of pegs was significantly greater at EOP +1 compared to Baseline $(P=0.001)$. For JTT times, the group $\times$ test $\times$ condition $\left(F_{(3,57)}=0.536, P=0.659, \eta^{2}=0.027\right)$, group $\times$ test $\left(F_{(3,57)}=0.228, P=0.876, \eta^{2}=0.012\right)$, and group $\times$ condition $\left(F_{(1,19)}=0.202, P=0.658, \eta^{2}=0.011\right)$ interactions were all non-statistically significant. However, there was a significant test $\times$ condition $\left(F_{(3,57)}=3.800, P=0.015, \eta^{2}=0.167\right.$;

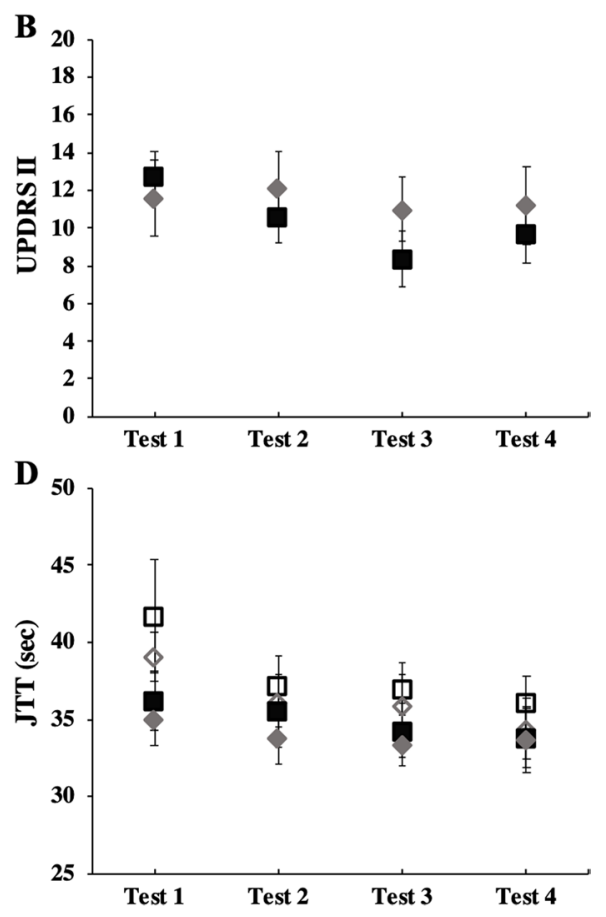

scores were similar for the c-tDCS and SHAM groups $(P=0.857)$ and the number of pegs was significantly greater at EOP +1 compared to Baseline $(P=0.001)$. PPT scores were significantly higher in the ON compared to the OFF state $(P=0.002)$. D. JTT times were similar for the c-tDCS and SHAM groups $(P=0.612)$ and JTT times were significantly lower at $\mathrm{EOP}+1, \mathrm{EOP}+14$, and $\mathrm{EOP}+28 \mathrm{com}-$ pared to Baseline $(P=0.023, P=0.003, P=0.001$ respectively). JTT times were significantly lower in the ON state compared to the OFF state $(P=0.001)$ 
Fig. 4D) interaction and post hoc analyses indicated that JTT times were significant lower in the $\mathrm{ON}$ state at Baseline, $\mathrm{EOT}+1$, and EOT +14 (all $P$ values $<0.009$ ). In addition, there was a significant main effect for test $\left(F_{(3,57)}=9.304\right.$, $P=0.001, \eta^{2}=0.329$ ) and post hoc analyses indicated that JTT times were significantly lower at $\mathrm{EOP}+1, \mathrm{EOP}+14$, and EOP +28 compared to Baseline $(P=0.023, P=0.003$, $P=0.001$ respectively). There was also a significant main effect for condition $\left(F_{(1,19)}=13.940, P=0.001, \eta^{2}=0.423\right)$, which indicated that JTT times were significantly lower in the ON state compared to the OFF state. Finally, the main effect for group was not statistically significant $\left(F_{(1,19)}=0.266, P=0.612, \eta^{2}=0.014\right)$.

\section{Transfer Tasks-Lower Extremity Tests}

For the step length, the group $\times$ test $\times$ condition $\left(F_{(3,57)}=1.097, P=0.358, \eta^{2}=0.055\right)$, group $\times$ test $\left(F_{(3,57)}=0.489, P=0.691, \eta^{2}=0.025\right)$, test $\times$ condition $\left(F_{(3,57)}=0.247, P=0.863, \eta^{2}=0.013\right)$, and group $\times$ condition $\left(F_{(1,19)}=0.362, P=0.555, \eta^{2}=0.019\right)$ interactions were all non-statistically significant. In addition, the main effect for group $\left(F_{(1,19)}=0.202, P=0.658, \eta^{2}=0.011\right)$, test $\left(F_{(3,57)}=0.275, P=0.843, \eta^{2}=0.014\right)$, and condition

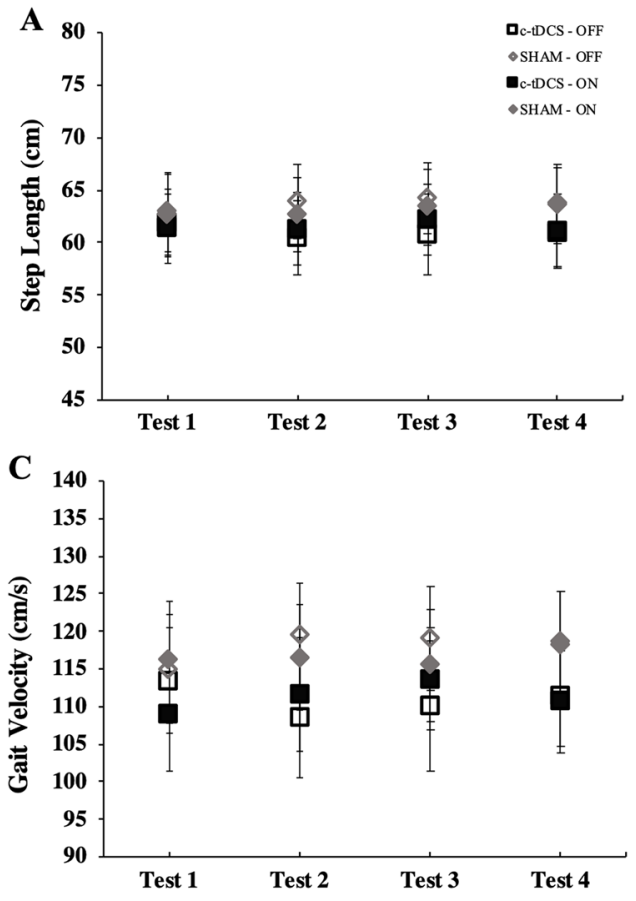

Fig. 5 Step length, step length CV, gait velocity, and balance composite score in the test sessions for the c-tDCS and SHAM groups in the ON and OFF states. A. Step length was similar for the c-tDCS and SHAM groups $(P=0.658)$, did not change across the test sessions $(P=0.843)$, and was similar in the ON and OFF states $(P=0.973)$. B. Step length $\mathrm{CV}$ was similar for the c-tDCS and SHAM groups $(P=0.745)$, did not change across the test sessions $(P=0.590)$, and
$\left(F_{(1,19)}=0.001, P=0.973, \eta^{2}=0.000\right)$ were not statistically significant (Fig. 5A). Similarly, the group $\times$ test $\times$ condition $\left(F_{(3,57)}=0.438, P=0.727, \eta^{2}=0.023\right)$, group $\times$ test $\left(F_{(3,57)}=0.551, P=0.650, \eta^{2}=0.028\right)$, test $\times$ condition $\left(F_{(3,57)}=0.188, P=0.904, \eta^{2}=0.010\right)$, and group $\times$ con dition $\left(F_{(1,19)}=0.659, P=0.427, \eta^{2}=0.034\right)$ interactions were all non- statistically significant for the Step Length CV (Fig. 5B). In addition, the main effect for group $\left(F_{(1,19)}=0.109, P=0.745, \eta^{2}=0.006\right)$, test $\left(F_{(3,57)}=0.644\right.$, $\left.P=0.590, \eta^{2}=0.033\right)$, and condition $\left(F_{(1,19)}=0.369\right.$, $\left.P=0.551, \eta^{2}=0.019\right)$ were not statistically significant. For gait velocity, there was a significant group $\times$ test $\times$ condition interaction $\left(F_{(3,57)}=3.954, P=0.019, \eta^{2}=0.159\right)$. Post hoc analyses indicated that the c-tDCS group had a lower gait velocity in the ON state at the baseline session $(P=0.021)$. Analogously, the SHAM group had a slower gait velocity in the ON state at the EOP +14 session $(P=0.039)$. Furthermore, the group $\times$ test $\left(F_{(3,57)}=0.399, P=0.754\right.$, $\left.\eta^{2}=0.021\right)$, test $\times$ condition $\left(\mathrm{F}_{(3,57)}=0.260, P=0.854\right.$, $\left.\eta^{2}=0.159\right)$, and group $\times$ condition $\left(F_{(1,19)}=0.259, P=0.616\right.$, $\left.\eta^{2}=0.013\right)$ interactions were all non-statistically significant. Finally, the main effect for $\operatorname{group}\left(F_{(1,19)}=0.402, P=0.533\right.$, $\left.\eta^{2}=0.021\right)$, test $\left(F_{(3,57)}=0.209, P=0.890, \eta^{2}=0.011\right)$, and condition $\left(F_{(1,19)}=0.056, P=0.816, \eta^{2}=0.003\right)$ were not

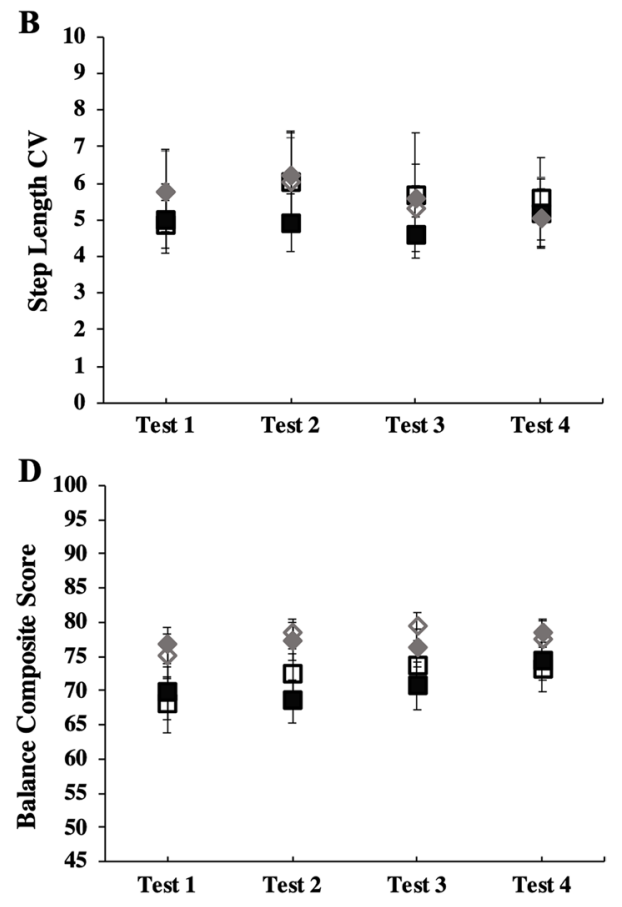

was similar in the $\mathrm{ON}$ and $\mathrm{OFF}$ states $(P=0.551)$. C. Gait velocity was similar for the c-tDCS and SHAM groups $(P=0.533)$, did not change across the test sessions $(P=0.890)$, and was similar in the $\mathrm{ON}$ and $\mathrm{OFF}$ states $(P=0.816)$. D. Balance composite score was similar for the c-tDCS and SHAM groups $(P=0.120)$, did not change across the test sessions, and was similar in the ON and OFF states $(P=0.717)$ 
statistically significant (Fig. 5C). For the balance composite score, the group $\times$ test $\times$ condition $\left(F_{(3,57)}=0.233, P=0.873\right.$, $\left.\eta^{2}=0.014\right)$, group $\times$ test $\left(F_{(3,57)}=0.851, P=0.472\right.$, $\left.\eta^{2}=0.028\right)$, and group $\times$ condition $\left(F_{(1,19)}=0.201, P=0.659\right.$, $\left.\eta^{2}=0.012\right)$ interactions were all non-statistically significant. However, the test $\times$ condition interaction was significant $\left(F_{(3,57)}=7.601, P=0.001, \eta^{2}=0.309\right)$, and post hoc analyses indicated that balance composite scores were higher in the OFF state at EOP $+14(P=0.012$, respectively). Similarly, there was a significant main effect for test $\left(F_{(3,57)}=4.331\right.$, $P=0.009,{ }^{2} 2=0.203$ ), but none of the post hoc comparisons were statistically significant (all $P$ values $>$ than 0.05 ). Finally, the main effect for $\operatorname{group}\left(F_{(1,19)}=0.325, P=0.120\right.$, $\left.\eta^{2}=0.130\right)$ and the main effect for condition $\left(F_{(1,19)}=0.136\right.$, $P=0.717, \eta^{2}=0.008$ ) were not significant (Fig. 5D).

\section{Practice Sessions}

The percent change (decline) in force error in the PGT from Practice Day 1 to Practice Day 9 was not statistically significantly $(P=0.698)$ different between the c-tDCS and SHAM groups (Fig. 3B). Similarly, the percent change (decline) in endpoint error in the AMT from Practice Day 1 to Practice Day 9 not significantly $(P=0.860)$ different between the c-tDCS and SHAM groups (Fig. 3D).

\section{Discussion}

The primary purpose was to determine the influence of long-term application of c-tDCS on motor learning in PD, whereas the secondary purpose was to examine the influence of long-term application of c-tDCS on transfer of motor learning in PD. The study produced six main findings: 1) the force error in the PGT significantly declined across the test sessions, but the reduction in force error was similar for the c-tDCS and SHAM groups; 2) the endpoint error in the AMT significantly declined across the test sessions, but the reduction in force error was similar for the c-tDCS and SHAM groups; 3) clinical rating scale scores (UPDRS-III and UPDRS-II) displayed no systematic changes across the test sessions and were not significantly different between the c-tDCS and SHAM groups; 4) the manual dexterity tests (PPT, JTT) improved slightly and to varying degrees across the tests sessions, but were not significantly different between the c-tDCS and SHAM groups; 5) lower extremity tests displayed no systematic changes across the test sessions and were not significantly different between the c-tDCS and SHAM groups; and 6) the UPDRS-III, PTT, and JTT were the only outcomes that exhibited improved scores in the ON state compared to the OFF state. These findings indicate that long-term application of c-tDCS concurrent with motor practice does not enhance motor learning to a greater extent than practice alone in PD. Similarly, long-term application of c-tDCS does not increase transfer of motor learning in PD. Collectively, these findings provide no evidence that c-tDCS applied repeatedly over multiple days is an effective intervention to improve motor learning in PD.

\section{Influence of c-tDCS on Motor Learning in Hand and Arm Tasks of the Upper Limb in PD}

Motor skill acquisition is a short-term, potentially temporary change in motor performance quantified during or in brief time periods (minutes to a few hours) during or after a practice session. Conversely, motor learning refers to a relatively long-term adaptation in motor performance that is usually quantified in retention tests in the days, weeks, or even months following practice. Furthermore, it has been shown that the processes of motor skill acquisition and learning involve different underlying physiological mechanisms realized over different time scales [26, 55]. A common criticism of many tDCS studies is that a single day of stimulation is insufficient to induce increases in motor learning and that repeated daily stimulation protocols are preferable due to possibility of M1-tDCS [11-13, 38] or c-tDCS [16] eliciting accumulating effects over multiple days or consolidation of motor learning.

Accordingly, the current study quantified motor learning in two practice tasks performed simultaneously with administration of c-tDCS across 9 days of practice as well as in retention tests performed 1, 14, and 28 days after practice ceased. It was originally hypothesized that c-tDCS would enhance motor learning in practice tasks to a greater degree than practice alone in PD. Contrary to this set of expectations, the improvements in motor learning in the practice tasks as indicated by significant reductions in force error in the PGT and endpoint error in the AMT across the test and practice sessions was similar between the c-tDCS and SHAM groups. Thus, c-tDCS did not significantly enhance the magnitude of motor learning exhibited in the practice task by individuals with PD. Furthermore, the practice task performance assessment results obtained in the test sessions revealed that the same pattern of results was present in both the $\mathrm{ON}$ and OFF medication states.

These negative outcomes contrast with the positive outcomes reported in previous single session c-tDCS studies in young and old adults $[15,17,19,20]$ as well as a study that applied c-tDCS for three consecutive days in young adults [16]. In particular, this includes a recent study in our laboratory [18], which found that c-tDCS significantly increased motor skill acquisition during a single day of practice and motor learning quantified in a retention test a day later in a very difficult overhand throwing task in young adults. 
The current results are also not consistent with the motor skill improvements observed in the majority of short-term M1-tDCS in PD [8-10, 34, 35]. Most notably, the results differ from a highly relevant study that applied M1-tDCS for 5 consecutive days in a cross-over design. Specifically, Valentino et al. (2014) reported significant reductions in the number and duration of freezing of gait episodes as well as UPDRS scores in PD at the end of the 5 stimulation sessions and at 2 and 4 weeks after stimulation had ended [14]. Conversely, a recent study in young adults found that c-tDCS did not increase whole body dynamic balance [56]. Furthermore, the present findings are in agreement with a study where a single administration of c-tDCS failed to improve scores in a clinical writing task in dystonia patients [57]. These results would seem to strongly support the current observations that c-tDCS may not be effective in augmenting motor skills in basal ganglia disorders, despite the fact that the cerebellum contributes to the motor impairments in these disorders [22, 23]. Taken together, the results of previous M1-tDCS studies in PD and the current findings indicate that targeting the cerebellum with tDCS may not be the most successful strategy to enhance motor skill acquisition and learning in PD.

\section{Influence of c-tDCS on Transfer of Motor Learning in PD}

A fundamental issue associated with motor learning and tDCS studies is whether performance improvements in a practice tasks can be generalized to non-practiced tasks. Any intervention will be of limited benefit if it is only applicable to a practice task as it is impractical to practice every impaired task in PD due to the myriad of motor tasks performed in daily activities. Despite the importance of the issue of transfer of motor learning, the number of studies that investigate transfer of motor learning relative to motor learning in general is exceedingly small. Accordingly, the influence of tDCS applied to any brain area in any population on transfer of motor learning has only been directly investigated in a handful of single session studies in young and older adults [15, 58, 59] and one long-term study in stroke [38], and these studies have provided contradictory findings.

Therefore, the current study employed 4 upper and 4 lower extremity transfer tasks that were evaluated over a time frame of several weeks. In addition, the upper extremity tasks had varying degrees of similarities with the practice tasks regarding the same vs different muscle groups, more complex versus less complex tasks, contraction types, and proximal vs distal control of the upper limb. Thus, the upper extremity transfer tasks could probably be assumed to represent different gradations of near transfer tasks, whereas the lower extremity tasks were obviously far transfer tasks. The results indicated that c-tDCS failed to improve performance of any of the transfer tasks in the c-tDCS group to a greater extent than in the SHAM group. More specifically, participants did improve their performance in a few of the transfer tasks (PTT, JTT, UPDRS-II) as evidenced by significant changes at various timepoints between the Baseline and EOP tests. However, the magnitude of these improvements did not differ between the c-tDCS and SHAM groups. Conversely, the remaining transfer tasks and, therefore, the majority displayed little modulation across the test sessions for either group, which also meant that c-tDCS did not produce any systematic effects on the transfer of motor learning. Thus, long-term c-tDCS did not improve transfer of motor learning in either far or near transfer tasks in the current study.

These findings differ from Orban de Xivre et al. (2011), which found that M1-tDCS induced generalization in some, but not all features of arm movement performance in young adults [58]. However, the present results are similar to a study in older adults that reported that performance augmentations in a trained task done concurrently with M1-tDCS did not translate to improved performance in two tasks involving the hand [59]. In addition, Block et al. (2013) found no evidence for transfer of adaptation learning due to c-tDCS, although the comparison in this study was unique as it was based on transfer to the opposite limb [15]. Furthermore, in another study that utilized nearly identical tasks to the current study, it was observed that application of M1-tDCS led to improvements in the accuracy of a pinch grip task did not generalize to either PPT or JTT scores in chronic stroke patients [38]. Taken together, the majority of the available evidence supports the current results and suggests that both M1-tDCS and c-tDCS exhibit a limited ability to induce transfer of motor learning in any study population including PD. Therefore, it appears that both M1-tDCS and c-tDCS primarily elicit task-specific modulatory effects that are only manifested when tDCS is applied in association with a particular motor task. Nonetheless, due to the paucity of research on the topic, there is clearly a need for a better understanding of the ability of tDCS to elicit improvements in the transfer of motor learning.

\section{Possible Factors Responsible for Failure of c-tDCS to Improve Motor Learning in PD}

The lack of ability of c-tDCS to improve motor skill and learning in arm and hand tasks in PD were unexpected findings based on the balance of the available literature. A close examination of review articles on motor skill acquisition and motor learning in M1-tDCS and c-tDCS studies in young and old adults [15-19] as well as M1-tDCS [7] in PD [8, 9] appears to reveal that about $75 \%$ of tDCS studies have shown positive effects. Thus, a smaller, but non-trivial number of 
studies are in accord with the present findings. Conversely, a higher percentage of long-term tDCS studies in the aforementioned populations appear to demonstrate positive results. Nonetheless, the contradictory findings between the current study and the majority of the literature implies that it should not be readily assumed that M1-tDCS or c-tDCS applied in the long-term always lead to enhancements in motor skill acquisition and learning. Accordingly, there are several possible factors responsible for the failure of c-tDCS to improve motor learning or transfer in PD.

The most likely explanation is due to the well-characterized imbalances in inhibitory and excitatory pathways from the cerebellum to M1, basal ganglia, and other cortical targets in PD compared to healthy young and older adults. Thus, c-tDCS administration in PD does not lead to the same positive motor outcomes as in healthy adults. For example, it has been clearly demonstrated using single and paired pulse TMS (transcranial magnetic stimulation) delivered to the cerebellum that at least one and likely several cerebellar-thalamic-cortical pathways provide inputs to different interneuronal populations in M1 [60-62]. For instance, the most commonly studied pathway involves a cerebellarthalamic-cortical tract that bifurcates out of the thalamus such that one pathway induces net inhibition on corticospinal output cells in M1, whereas the other pathway induces net facilitation (see Fig. 6 in Reis et al. 2008) [60]. Furthermore, another TMS study that used anterior-posterior and posterior-anterior directed TMS currents, which have been shown to activate two different subsets of interneurons in M1, demonstrated that these two neuronal populations receive inputs from separate cerebellar pathways [61]. Most importantly, previous studies have provided evidence that when single TMS pulses, repetitive TMS, and c-tDCS are applied to the cerebellum they are not selective enough to activate these or any additional cerebellar pathways individually. Therefore, it is plausible that the widespread alterations, dysfunction, and compensatory processes present in these cerebellar-M1 pathways in PD could have contributed to the lack of c-tDCS effects in the current study. Finally, analogous processes would likely occur in the recently identified pathways between the cerebellum and basal ganglia, which also ultimately impact the output cells of M1 through the basal ganglia-cortico loop. Taken together, the extensive system-wide network dysfunctions in numerous neural circuits between cerebellum, basal ganglia, and M1 may have led to the lack of positive c-tDCS effects on motor learning in PD.

Another plausible explanation could be that two weeks of c-tDCS application may not be sufficient to substantially improve motor learning in PD. However, this is generally a more common criticism of single day tDCS studies that do not find positive results in movement disorders. For instance, it was argued justifiably in an acute c-tDCS study, which failed to enhance writing skills in dystonia, that one c-tDCS session is unlikely to be adequate to supersede years of disease processes [57]. However, this criticism is rarely directed toward multi-day tDCS studies, despite the obvious observation that 3-10 stimulation sessions over 1-2 weeks is a very short time span relative to the time disease processes have been consolidating for years. Perhaps this is due to the fact that the vast majority of long-term M1-tDCS [11-13] a long-term c-tDCS study in young adults [16], as well as a 2-week study in PD [14], have all reported large increases in motor learning. Nonetheless, the relatively short stimulation period of the current study relative to the time most PD patients have had the disease may not have been long enough for c-tDCS to enhance motor learning. Accordingly, what is considered a long-term tDCS study is not a long time period relative to the time since diagnosis for most individuals with PD as to our knowledge no tDCS studies in the motor system have applied stimulation for more than 10 sessions. In conclusion, it is possible that longer time periods of $\mathrm{c}-\mathrm{tDCS}$ administration could evoke improvements in motor learning in PD, but this explanation is very unlikely.

An additional explanation is that the parameters of c-tDCS (montage size and position, current strength, stimulation duration per session) or motor tasks utilized were not optimal. Accordingly, a number of previous c-tDCS studies in healthy adults have attained positive results with slightly different combinations of c-tDCS parameters [63, 64]. While this proposition is possible, it appears to be highly improbable as the current study used identical c-tDCS parameters to whose that elicited large motor skill and learning enhancements in a previous study in our lab [18] and in many other c-tDCS studies from another research group [15-17, 19, 20]. It is also highly doubtful that the practice tasks chosen in the present study were not ideal as numerous studies have demonstrated that these tasks are characterized by high cerebellar involvement $[45,48-50]$ and are highly responsive to tDCS $[12,13,16,38,52,65,66]$.

A final possible factor for the lack of c-tDCS effects could due to the interindividual variability in the responses to tDCS that are thought to be mediated by a combination of biological, anatomical, and physiological factors [67]. These factors likely lead to differences in the amount of tDCS current reaching the brain and the magnitude of behavioral responses. The cerebellum could be especially subject to individual differences in these aspects due to the variation in nerve fiber orientation and convoluted nature of the cerebellar cortex [29]. These findings are supported by several neuroimaging studies. In regard to general activation, two studies displayed different results in that anodal c-tDCS significantly increased cerebellar activation in one study [68], whereas anodal c-tDCS did not increase cerebellar cortex or dentate activation in another study [69]. This was despite the $\mathrm{c}-\mathrm{tDCS}$ parameters being very similar, but not identical, 
across studies and in the current study. Furthermore, a magnetic resonance spectroscopy study found large individual variability in cerebellar gamma-aminobutyric acid (GABA) and glutamate (GLU) in response to c-tDCS [70]. At the same time, group level analyses found no significant differences in visuomotor task performance or GABA and GLU levels during stimulation between the c-tDCS and SHAM groups. However, c-tDCS enhanced performance in the motor task during a retention test, but only in a sub-group of participants who displayed decreased cerebellar GLU levels. Apparent interindividual differences in responses to c-tDCS were also seen by Liebrand et al. (2020) as sequence learning was improved in a group who received c-tDCS [71]. This behavioral improvement occurred concomitant with a significant decrease in learning-specific negative modulation of putamen to cerebellar connections as revealed by fMRI. However, there was no correlation between the increase in behavioral performance and the changes in the putamen to cerebellar connections. These neuroimaging results are supported by a series of recent studies (reviewed in [29]) where several different research groups have not been able to replicate their own previous studies that found enhancements of motor performance due to c-tDCS. Finally, the absence of improvements in motor learning in the present study could be due to some combination of the factors described above and these possibilities should be addressed in subsequent research.

\section{Limitations}

Despite the clear findings that c-tDCS did not enhance motor learning in PD, the study had various limitations that should be acknowledged. The major limitation was the relatively small sample size. This may have precluded the ability to detect significant effects due to c-tDCS. However, the sample size was close to the average tDCS motor skill study, which appears to be 13 (see tables of Buch et al. [7]). It could also be argued that the low sample size issue is somewhat mitigated based on the futility analysis results and because multiple days of tDCS usually leads to large, accumulating effects $[12,13,16,38]$. Thus, if c-tDCS would have elicited positive effects, they most likely would have been apparent over the 9 days of stimulation. Another limitation of the study was that most of participants were Hoehn and Yahr Stage 2. Thus, it is possible that c-tDCS could have been effective if only more or less affected individuals with PD were enrolled. However, this could also have led to ceiling or floor effects.

The study was also subject to general limitations inherent to tDCS. For instance, some research has shown that a large percentage of the current may not reach the brain area of interest [72]. Similarly, a few studies have found that
c-tDCS causes only superficial, focal excitability changes in the cerebellum and in M1 [73, 74]. This is consistent with a recent meta-analysis [75] which concluded that the available studies show mixed findings on the influence of c-tDCS on M1 excitability. Specifically, some studies observed a slight increase in MEPs, whereas others found a slight decrease. The overall results of the meta-analysis indicated that the influence of c-tDCS on MEP amplitude was non-significant [75]. This is most likely because c-tDCS activates both inhibitory and excitatory circuits in M1 [76, 77], which may lead to a lack of observeable changes in global measures of M1 excitability such as resting motor threshold, recruitment curves, and MEPs. In addition, it has been established that increases in MEP amplitudes induced by tDCS of M1 are not correlated with the amount of motor learning [78]. This implies that when enhancements in motor learning are observed following c-tDCS, they are likely disassociated from any MEP changes induced in M1. Another possible limitation was identified in a recent study, which found that the effects of a related method of transcranial electrical stimulation were due to the transcutaneous stimulation of peripheral nerves [79]. Theoretically, if this were to also be true for tDCS, complex consequences could result that could attenuate, interfere, or even be responsible for the normally observed tDCS effects. Finally, other forms of non-invasive brain stimulation that are more focal or have effects that are mediated through different physiological mechanisms compared with tDCS could be more effective in PD. For instance, transcranial alternating current stimulation (tACS) of the cerebellum can also improve motor performance in healthy adults [80] and has the potential advantage of being able to induce entrainment at specified frequencies between different brain regions. This differs from the actions of tDCS where a certain brain area is facilitated or inhibited. Accordingly, accumulating evidence has underscored the importance of abnormal network oscillations (oscillopathies) in neurodegenerative diseases, especially PD [81]. All of these issues and limitations need to be addressed in future studies.

\section{Conclusions}

In summary, the long-term application of c-tDCS simultaneous with motor practice did not enhance motor learning of hand and arm motor tasks to a greater extent than practice alone in PD. In addition, the long-term application of c-tDCS did not increase transfer of motor learning to clinical rating scales with motor components, manual dexterity tasks involving the hand and arm, or lower extremity gait and balance tasks in PD. Collectively, these findings provide no evidence that c-tDCS applied repeatedly over multiple days is an effective intervention to improve motor learning in PD. 
Future studies, should probably focus on the examination of long-term administration of tDCS to cortical areas such as dorsolateral prefrontal cortex, premotor cortex, supplementary motor area, and especially M1 to improve motor learning in PD.

Acknowledgements This research was supported by a Mountain West Clinical Translational Research-Infrastructure Network (CTRIN IDEA), NIGMS, NIH, Grant \#U54GM104944. The authors would also like to thank Kathy Nagle for her help with the study.

\section{Declarations}

Ethics Approval and Informed Consent The study protocols were approved by the institutional review board at the University of Nevada Las Vegas All participants signed a written informed consent and were treated in accordance with the Declaration of Helsinki.

Conflict of Interest The authors declare no competing interests.

\section{References}

1. Chen JJ. Parkinson's disease: health-related quality of life, economic cost, and implications of early treatment. Am J Manag Care. 2010;16 Suppl Implications:S87-93

2. Bernheimer H, Birkmayer W, Hornykiewicz O, Jellinger K, Seitelberger F. Brain dopamine and the syndromes of Parkinson and Huntington. Clinical, morphological and neurochemical correlations. J Neurol Sci. 1973;20(4):415-55.

3. Archer T, Fredriksson A, Johansson B. Exercise alleviates Parkinsonism: clinical and laboratory evidence. Acta Neurol Scand. 2011;123(2):73-84.

4. Morris ME, Huxham F, McGinley J, Dodd K, Iansek R. The biomechanics and motor control of gait in Parkinson disease. Clin Biomech (Bristol, Avon). 2001;16(6):459-70.

5. Brunoni AR, Nitsche MA, Bolognini N, Bikson M, Wagner T, Merabet L, et al. Clinical research with transcranial direct current stimulation (tDCS): Challenges and future directions. Brain Stimul. 2012;5(3):175-95.

6. Nitsche MA, Cohen LG, Wassermann EM, Priori A, Lang N, Antal A, et al. Transcranial direct current stimulation: State of the art 2008. Brain Stimul. 2008;1(3):206-23.

7. Buch ER, Santarnecchi E, Antal A, Born J, Celnik PA, Classen $\mathrm{J}$, et al. Effects of tDCS on motor learning and memory formation: A consensus and critical position paper. Clin Neurophysiol. 2017;128(4):589-603.

8. Beretta VS, Conceicao NR, Nobrega-Sousa P, Orcioli-Silva D, Dantas L, Gobbi LTB, et al. Transcranial direct current stimulation combined with physical or cognitive training in people with Parkinson's disease: a systematic review. J Neuroeng Rehabil. 2020;17(1):74.

9. Simpson MW, Mak M. The effect of transcranial direct current stimulation on upper limb motor performance in Parkinson's disease: a systematic review. J Neurol. 2019.

10. Broeder S, Nackaerts E, Heremans E, Vervoort G, Meesen R, Verheyden G, et al. Transcranial direct current stimulation in Parkinson's disease: Neurophysiological mechanisms and behavioral effects. Neurosci Biobehav Rev. 2015;57:105-17.
11. Prichard G, Weiller C, Fritsch B, Reis J. Effects of different electrical brain stimulation protocols on subcomponents of motor skill learning. Brain Stimul. 2014;7(4):532-40.

12. Reis J, Fischer JT, Prichard G, Weiller C, Cohen LG, Fritsch B. Time- but not sleep-dependent consolidation of tDCS-enhanced visuomotor skills. Cereb Cortex. 2013;25(1):109-17.

13. Reis J, Schambra HM, Cohen LG, Buch ER, Fritsch B, Zarahn E, et al. Noninvasive cortical stimulation enhances motor skill acquisition over multiple days through an effect on consolidation. Proc Natl Acad Sci U S A. 2009;106(5):1590-5.

14. Valentino F, Cosentino G, Brighina F, Pozzi NG, Sandrini G, Fierro B, et al. Transcranial direct current stimulation for treatment of freezing of gait: a cross-over study. Mov Disord. 2014;29(8):1064-9.

15. Block H, Celnik P. Stimulating the cerebellum affects visuomotor adaptation but not intermanual transfer of learning. Cerebellum. 2013;12(6):781-93.

16. Cantarero G, Spampinato D, Reis J, Ajagbe L, Thompson T, Kulkarni K, et al. Cerebellar direct current stimulation enhances on-line motor skill acquisition through an effect on accuracy. J Neurosci. 2015;35(7):3285-90.

17. Galea JM, Vazquez A, Pasricha N, de Xivry JJ, Celnik P. Dissociating the roles of the cerebellum and motor cortex during adaptive learning: the motor cortex retains what the cerebellum learns. Cereb Cortex. 2011;21(8):1761-70.

18. Jackson AK, de Albuquerque LL, Pantovic M, Fischer KM, Guadagnoli MA, Riley ZA, et al. Cerebellar transcranial direct current stimulation enhances motor learning in a complex overhand throwing task. Cerebellum. 2019;18(4):813-6.

19. Jayaram G, Tang B, Pallegadda R, Vasudevan EV, Celnik P, Bastian A. Modulating locomotor adaptation with cerebellar stimulation. J Neurophysiol. 2012;107(11):2950-7.

20. Hardwick RM, Celnik PA. Cerebellar direct current stimulation enhances motor learning in older adults. Neurobiol Aging. 2014;35(10):2217-21.

21. Boisgontier MP. Motor aging results from cerebellar neuron death. Trends Neurosci. 2015;38(3):127-8.

22. Ni Z, Pinto AD, Lang AE, Chen R. Involvement of the cerebellothalamocortical pathway in Parkinson disease. Ann Neurol. 2010;68(6):816-24.

23. Wu T, Hallett M. The cerebellum in Parkinson's disease. Brain. 2013;136(Pt 3):696-709.

24. Bostan AC, Dum RP, Strick PL. The basal ganglia communicate with the cerebellum. Proc Natl Acad Sci U S A. 2010;107(18):8452-6.

25. Li H, Lei X, Yan T, Li H, Huang B, Li L, et al. The temporary and accumulated effects of transcranial direct current stimulation for the treatment of advanced Parkinson's disease monkeys. Sci Rep. 2015;5:12178.

26. Krakauer JW, Mazzoni P. Human sensorimotor learning: adaptation, skill, and beyond. Curr opin neurobiol. 2011;21(4):636-44.

27. Celnik P. Understanding and modulating motor learning with cerebellar stimulation. Cerebellum. 2015;14(2):171-4.

28. Grimaldi G, Argyropoulos GP, Boehringer A, Celnik P, Edwards MJ, Ferrucci R, et al. Non-invasive cerebellar stimulation-a consensus paper. Cerebellum. 2014;13(1):121-38.

29. Miterko LN, Baker KB, Beckinghausen J, Bradnam LV, Cheng MY, Cooperrider J, et al. Consensus paper: experimental neurostimulation of the cerebellum. Cerebellum. 2019;18(6):1064-97.

30. Spampinato D, Celnik P. Temporal dynamics of cerebellar and motor cortex physiological processes during motor skill learning. Sci Rep. 2017;7:40715.

31. Abbruzzese G, Trompetto C, Marinelli L. The rationale for motor learning in Parkinson's disease. Eur J Phys Rehabil Med. 2009;45(2):209-14. 
32. Agostino R, Curra A, Soldati G, Dinapoli L, Chiacchiari L, Modugno N, et al. Prolonged practice is of scarce benefit in improving motor performance in Parkinson's disease. Mov Disord. 2004;19(11):1285-93.

33. Wu T, Liu J, Zhang H, Hallett M, Zheng Z, Chan P. Attention to automatic movements in Parkinson's disease: modified automatic mode in the striatum. Cereb Cortex. 2015;25(10):3330-42.

34. Boggio PS, Castro LO, Savagim EA, Braite R, Cruz VC, Rocha RR, et al. Enhancement of non-dominant hand motor function by anodal transcranial direct current stimulation. Neurosci Lett. 2006;404(1-2):232-6.

35. Fregni F, Boggio PS, Santos MC, Lima M, Vieira AL, Rigonatti SP, et al. Noninvasive cortical stimulation with transcranial direct current stimulation in Parkinson's disease. Mov Disord. 2006;21(10):1693-702.

36. Oldfield RC. The assessment and analysis of handedness: the Edinburgh inventory. Neuropsychologia. 1971;9(1):97-113.

37. Defer GL, Widner H, Marie RM, Remy P, Levivier M. Core assessment program for surgical interventional therapies in Parkinson's disease (CAPSIT-PD). Mov Disord. 1999;14(4):572-84.

38. Hamoudi M, Schambra HM, Fritsch B, Schoechlin-Marx A, Weiller C, Cohen LG, et al. Transcranial direct current stimulation enhances motor skill learning but not generalization in chronic stroke. Neurorehabil Neural Repair. 2018;32(4-5):295-308.

39. Danna-Dos Santos A, Poston B, Jesunathadas M, Bobich LR, Hamm TM, Santello M. Influence of fatigue on hand muscle coordination and EMG-EMG coherence during three-digit grasping. J Neurophysiol. 2010;104(6):3576-87.

40. Poston B, Danna-Dos Santos A, Jesunathadas M, Hamm TM, Santello M. Force-independent distribution of correlated neural inputs to hand muscles during three-digit grasping. J Neurophysiol. 2010;104(2):1141-54.

41. Poston B, Christou EA, Enoka JA, Enoka RM. Timing variability and not force variability predicts the endpoint accuracy of fast and slow isometric contractions. Exp Brain Res. 2010;202(1):189-202

42. Voelcker-Rehage C, Alberts JL. Age-related changes in grasping force modulation. Exp Brain Res. 2005;166(1):61-70.

43. Voelcker-Rehage C, Alberts JL. Effect of motor practice on dualtask performance in older adults. J Gerontol B Psychol Sci Soc Sci. 2007;62(3):P141-8.

44. Pradhan SD, Brewer BR, Carvell GE, Sparto PJ, Delitto A, Matsuoka Y. Assessment of fine motor control in individuals with Parkinson's disease using force tracking with a secondary cognitive task. J Neurol Phys Ther. 2010;34(1):32-40.

45. Vaillancourt DE, Thulborn KR, Corcos DM. Neural basis for the processes that underlie visually guided and internally guided force control in humans. J Neurophysiol. 2003;90(5):3330-40.

46. Seidler RD, Alberts JL, Stelmach GE. Multijoint movement control in Parkinson's disease. Exp Brain Res. 2001;140(3):335-44.

47. Poston B, Van Gemmert AW, Sharma S, Chakrabarti S, Zavaremi $\mathrm{SH}$, Stelmach G. Movement trajectory smoothness is not associated with the endpoint accuracy of rapid multi-joint arm movements in young and older adults. Acta Psychol (Amst). 2013;143(2):157-67.

48. Bastian AJ, Martin TA, Keating JG, Thach WT. Cerebellar ataxia: abnormal control of interaction torques across multiple joints. $\mathrm{J}$ Neurophysiol. 1996;76(1):492-509.

49. Timmann D, Lee P, Watts S, Hore J. Kinematics of arm joint rotations in cerebellar and unskilled subjects associated with the inability to throw fast. Cerebellum. 2008;7(3):366-78.

50. Flament D, Hore J. Movement and electromyographic disorders associated with cerebellar dysmetria. J Neurophysiol. 1986;55(6):1221-33.
51. Tseng YW, Diedrichsen J, Krakauer JW, Shadmehr R, Bastian AJ. Sensory prediction errors drive cerebellum-dependent adaptation of reaching. J Neurophysiol. 2007;98(1):54-62.

52. Hummel FC, Heise K, Celnik P, Floel A, Gerloff C, Cohen LG. Facilitating skilled right hand motor function in older subjects by anodal polarization over the left primary motor cortex. Neurobiol Aging. 2010;31(12):2160-8.

53. Galea JM, Jayaram G, Ajagbe L, Celnik P. Modulation of cerebellar excitability by polarity-specific noninvasive direct current stimulation. J Neurosci. 2009;29(28):9115-22.

54. Albuquerque LL, Fischer KM, Pauls AL, Pantovic M, Guadagnoli MA, Riley ZA, et al. An acute application of transcranial random noise stimulation does not enhance motor skill acquisition or retention in a golf putting task. Hum Mov Sci. 2019;66:241-8.

55. Bologna M, Rocchi L, Paparella G, Nardella A, Li Voti P, Conte A, et al. Reversal of practice-related effects on corticospinal excitability has no immediate effect on behavioral outcome. Brain Stimul. 2015;8(3):603-12.

56. Steiner KM, Enders A, Thier W, Batsikadze G, Ludolph N, Ilg $\mathrm{W}$, et al. Cerebellar tDCS does not improve learning in a complex whole body dynamic balance task in young healthy subjects. PLoS One. 2016;11(9):e0163598.

57. Sadnicka A, Hamada M, Bhatia KP, Rothwell JC, Edwards MJ Cerebellar stimulation fails to modulate motor cortex plasticity in writing dystonia. Mov Disord. 2014;29(10):1304-7.

58 Orban de Xivry JJ, Marko MK, Pekny SE, Pastor D, Izawa J, Celnik P, et al. Stimulation of the human motor cortex alters generalization patterns of motor learning. J Neurosci. 2011;31(19):7102-10.

59. Parikh PJ, Cole KJ. Effects of transcranial direct current stimulation in combination with motor practice on dexterous grasping and manipulation in healthy older adults. Physiol Rep. 2014;2(3):e00255.

60. Reis J, Swayne OB, Vandermeeren Y, Camus M, Dimyan MA, Harris-Love M, et al. Contribution of transcranial magnetic stimulation to the understanding of cortical mechanisms involved in motor control. J Physiol. 2008;586(2):325-51.

61. Spampinato DA, Celnik PA, Rothwell JC. Cerebellar-motor cortex connectivity: one or two different networks? J Neurosci. 2020;40(21):4230-9.

62. Hamada M, Galea JM, Di Lazzaro V, Mazzone P, Ziemann U, Rothwell JC. Two distinct interneuron circuits in human motor cortex are linked to different subsets of physiological and behavioral plasticity. J Neurosci. 2014;34(38):12837-49.

63. Kenville R, Maudrich T, Maudrich D, Villringer A, Ragert P. Cerebellar transcranial direct current stimulation improves maximum isometric force production during isometric barbell squats. Brain Sci. 2020;10(4).

64. Oldrati V, Schutter D. Targeting the human cerebellum with transcranial direct current stimulation to modulate behavior: a metaanalysis. Cerebellum. 2018;17(2):228-36.

65. Hummel F, Celnik P, Giraux P, Floel A, Wu WH, Gerloff C, et al. Effects of non-invasive cortical stimulation on skilled motor function in chronic stroke. Brain. 2005;128(Pt 3):490-9.

66. Hummel F, Cohen LG. Improvement of motor function with noninvasive cortical stimulation in a patient with chronic stroke. Neurorehabil Neural Repair. 2005;19(1):14-9.

67. Pellegrini M, Zoghi M, Jaberzadeh S. Biological and anatomical factors influencing interindividual variability to noninvasive brain stimulation of the primary motor cortex: a systematic review and meta-analysis. Rev Neurosci. 2018;29(2):199-222.

68. Macher K, Bohringer A, Villringer A, Pleger B. Cerebellarparietal connections underpin phonological storage. J Neurosci. 2014;34(14):5029-37.

69. Kuper M, Mallick JS, Ernst T, Kraff O, Thurling M, Stefanescu MR, et al. Cerebellar transcranial direct current stimulation 
modulates the fMRI signal in the cerebellar nuclei in a simple motor task. Brain Stimul. 2019;12(5):1169-76.

70. Jalali R, Chowdhury A, Wilson M, Miall RC, Galea JM. Neural changes associated with cerebellar tDCS studied using MR spectroscopy. Exp Brain Res. 2018;236(4):997-1006.

71. Liebrand M, Karabanov A, Antonenko D, Floel A, Siebner HR, Classen J, et al. Beneficial effects of cerebellar tDCS on motor learning are associated with altered putamen-cerebellar connectivity: A simultaneous tDCS-fMRI study. NeuroImage. 2020;223:117363.

72. Voroslakos M, Takeuchi Y, Brinyiczki K, Zombori T, Oliva A, Fernandez-Ruiz A, et al. Direct effects of transcranial electric stimulation on brain circuits in rats and humans. Nature Commun. 2018;9(1):483.

73. Neuling T, Wagner S, Wolters $\mathrm{CH}$, Zaehle T, Herrmann CS. Finite-element model predicts current density distribution for clincal applications of tDCS and tACS. Front Psychiatry. 2012;3(83):1-10.

74. Rampersad SM, Janssen AM, Lucka F, Aydin U, Lanfer B, Lew $\mathrm{S}$, et al. Simulating transcranial direct current stimulation with a detailed anisotropic human head model. IEEE Trans Neural Syst Rehabil Eng. 2014;22(3):441-52.

75. Behrangrad S, Zoghi M, Kidgell D, Jaberzadeh S. Does cerebellar non-invasive stimulation affect corticospinal excitability in healthy individuals? A systematic review of literature and metaanalysis. Neurosci Lett. 2019;706:128-39.
76. Ates MP, Alaydin HC, Cengiz B. The effect of the anodal transcranial direct current stimulation over the cerebellum on the motor cortex excitability. Brain Res Bull. 2018;140:114-9.

77. Opie GM, Liao WY, Semmler JG. Interactions between cerebellum and the intracortical excitatory circuits of motor cortex: a mini-review. Cerebellum. 2021.

78. Lopez-Alonzo V, Cheeran B, Fernandez-del-Olmo M. Relationship between non-invasive brain stimulation-induced plasticity and capacity for motor learning. Brain Stimul. 2015;8(6):1209-19.

79. Asamoah B, Khatoun A, Mc LM. tACS motor system effects can be caused by transcutaneous stimulation of peripheral nerves. Nat Commun. 2019;10(1):266.

80. Naro A, Bramanti A, Leo A, Manuli A, Sciarrone F, Russo M, et al. Effects of cerebellar transcranial alternating current stimulation on motor cortex excitability and motor function. Brain Struct Funct. 2017;222(6):2891-906.

81. Nimmrich V, Draguhn A, Axmacher N. Neuronal network oscillations in neurodegenerative diseases. Neuromolecular Med. 2015;17(3):270-84.

Publisher's Note Springer Nature remains neutral with regard to jurisdictional claims in published maps and institutional affiliations. 